\title{
Study on the Influence of Radon Collector Parame
}

\author{
Shangting Jiang, Jian Shan*, Hui Yang, Jinglin Li, Songsong Li, Tao Guo \\ Institute of Nuclear Science and Technology, Nan Hua University, Hengyang, China \\ Email address: \\ 466746962@qq.com (Shangting Jiang), shanjian0666@163.com (Jian Shan),1030667741@qq.com (Hui Yang), \\ 6710010019@qq.com (Jinglin Li),757420980@qq.com (Songsong Li),1532103928@qq.com (Tao Guo) \\ ${ }^{*}$ Corresponding author
}

\section{To cite this article:}

Shangting Jiang, Jian Shan, Hui Yang, Jinglin Li, Songsong Li, Tao Guo. Study on the Influence of Radon Collector Parame. American Journal of Physics and Applications. Vol. 7, No. 4, 2019, pp. 109-117. doi: 10.11648/j.ajpa.20190704.13

Received: July 22, 2019; Accepted: August 14, 2019; Published: September 2, 2019

\begin{abstract}
Accurate measurement of radon exhalation rate of building materials plays an important role in controlling indoor radon concentration. In order to achieve rapid and accurate measurement of radon exhalation, the influence of the volume, base area and pumping flow rate of radon collector on radon exhalation rate was studied to optimize the measurement parameters of radon exhalation rate and improve the measurement efficiency of radon exhalation rate. The study has shown that the larger the volume of radon collector is, the longer the radon concentration equilibrium time will be when radon exhalation rate is measured with constant pumping flow rate and surface precipitation rate, while the influence of the volume of radon collector on the equilibrium radon concentration can be neglected, but there is a specific linear relationship between the equilibrium radon concentration and the base area of radon collector. When the radon exhalation rate is measured with constant volume and base area of radon collector, the higher the pumping flow rate is, the shorter the radon concentration equilibrium time is and the smaller the equilibrium radon concentration is. When the radon exhalation rate is $3.9 \mathrm{~Bq} \cdot \mathrm{m}^{-2} \cdot \mathrm{s}^{-1}$ in the experiment, the optimum volume of radon collector is $2.1 \times 10^{-3} \mathrm{~m}^{3}$, the optimum base area is $3.46 \times 10^{-2} \mathrm{~m}^{-2}$, and the optimum pumping flow rate is $1.349 \times 10^{-5} \mathrm{~m}^{3} / \mathrm{s}$. The measurement parameters of the radon exhalation rate, such as the best volume and base area of radon collector and the pumping flow rate can be obtained for different radon exhalation rates through this optimization method.
\end{abstract}

Keywords: Radon Exhalation Rate, MATLAB Simulation, Radon Collector, Pumping Flow Rate

\section{Introduction}

Radon exhalation rate is one of the most important factors determining the radon concentration in a certain environment, while radon and its daughters have always been the main contributors to the natural radiation dose for human. According to the UNSCEAR 2000 report, the annual effective dose of natural radiation from radon inhalation and its daughters to people in normal background areas of the world is $1.3 \mathrm{mSv}$ on average, accounting for $54.2 \%$ [1-2] of the annual effective dose of natural radiation to adults worldwide from natural ionizing radiation, which is $2.4 \mathrm{mSv}$, Studies have shown that indoor radon radiation to human body accounts for $90 \%$ of the total radon radiation to human body [3]. At present, the radon radiation to Chinese people mainly comes from the obvious increase of radon exhalation rate in buildings, so it is of particular significance to measure the radon exhalation rate of building materials accurately
[4-8]. Radon exhalation rate measurement is usually conducted by attaching a well-sealed radon collector to the surface of the medium, which accumulates radon separated out from the surface of the medium, thus the radon exhalation rate on the surface of the medium can be calculated by measuring radon concentration. Although there are many studies at home and abroad on the measurement of radon exhalation rate on the surface of medium [9-13], there are few studies [14-16] on the influence of radon collector volume, base area and pumping flow rate on the radon exhalation rate on the surface of medium, while the determination of relevant parameters of radon collector is of great importance for the accurate measurement of radon exhalation rate. In this study, firstly, theoretical calculation was carried out and the simulation function of MATLAB was used to simulate and calculate some parameters in the process of radon exhalation rate measurement. Then, corresponding experiment was performed on the reference 
standard device of radon exhalation rate for verification through double-chamber open-loop circulation mode by using the RAD7 radon detector. Through theoretical calculation and practical measurement, the optimization of equilibrium radon concentration and radon concentration equilibrium time in radon collector volume, base area, pumping flow rate and radon exhalation rate measurement were discussed.

\section{Principles and Methods}

\subsection{Measurement of Radon Exhalation Rate by Double Chamber Open-Loop Circulation Method}

The radon collector was buckled upside-down on the surface of the medium to be measured with fresh air continuously pumped from outside through the external pump (compared with the radon concentration in the equilibrium state in radon collector, the gas containing radon can be neglected) into the radon collector whose air outlet. is connected to the radon detector through a drying pipe. The detailed process of the radon measurement system is shown in Figure 1.

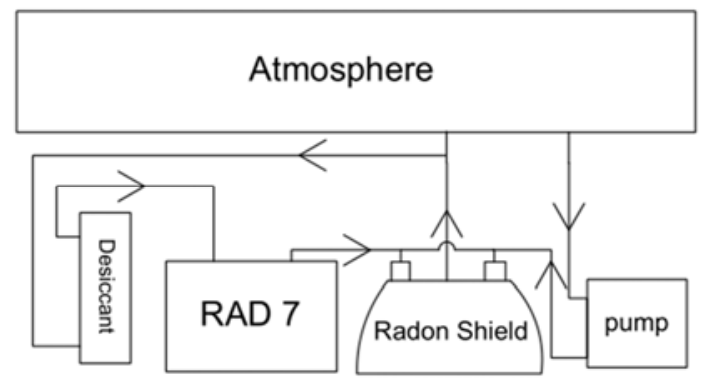

Figure 1. Radon Exhalation Rate Measuring Device.

The physical experimental device is shown in Figure 2. The radon collector is upside down on the surface of the measured medium, and then the air outlet of the radon collector is connected with a Tee- joint with one of the outlet of the joint connected with the dry pipe before connecting with the air inlet of the measuring instrument (RAD7 radon detector was selected in this experiment), and the other outlet of the Tee-joint is connected with the external atmosphere. Four air inlets of the radon collector are connected with the outlet of the external flow pump through three Tee-joints and the inlet of the external flow rate pump is directly connected with the clean atmosphere outside. Radon exhalation rate on the surface of medium can be calculated by measuring radon concentration in radon collector with radon detector.

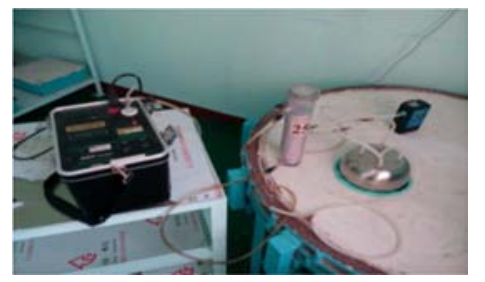

Figure 2. Physical diagram of the experimental device.
Under this system, the variation of radon concentration in radon collector is described by equation (1) [9]:

$$
\frac{d c}{d t}=\frac{P S}{V}-\frac{L c}{V}-(\lambda+R) c
$$

Among them, $\mathrm{P}$ is the radon exhalation rate of the measured medium surface, $\mathrm{Bq} \cdot \mathrm{m}^{-2} \cdot \mathrm{s}^{-1} ; \mathrm{S}$ is the base area of radon collector, $\mathrm{m}^{2}$; $\mathrm{L}$ is the flow of the pump, $\mathrm{m}^{3} / \mathrm{s} ; \mathrm{V}$ is the space volume of radon collector, $\mathrm{m}^{3} ; \mathrm{C}$ is the radon concentration in radon collector after accumulation of $\mathrm{t}$ (time), that is, the equilibrium radon concentration $\mathrm{Bq} \bullet \mathrm{m}^{-3} ; \lambda$ is the decay constant of radon, $\mathrm{s}^{-1} ; \mathrm{R}$ is the leakage and anti-diffusion rate of radon; $t$ is the equilibrium time of radon concentration, $s$.

In the measurement, the pumping flow rate $\mathrm{L}$ is adjusted to ensure that radon concentration in radon collector is not too high. Meanwhile, the sealing material is used to ensure that the radon collector is well-sealed, so that the factors of leakage and anti-diffusion in radon collector can be ignored. That is to say, when $\mathrm{L} / \mathrm{V}>>\lambda+\mathrm{R}$, Formula (1) can be simplified as follows:

$$
\frac{d c}{d t}=\frac{P S-L c}{V}
$$

The radon concentration in radon collector is made zero at the initial time, and the variation of radon concentration in radon collector is expressed by the following formula:

$$
c(\mathrm{t})=\frac{P S\left(1-e^{-L t / V}\right)}{L}
$$

Among them, $\mathrm{c}(\mathrm{t})$ is radon concentration at $\mathrm{t}$ time, $\mathrm{Bq} / \mathrm{m}^{3}$; $\mathrm{t}$ is time of collecting radon in radon collector, $\mathrm{s}$; $\mathrm{P}$ is radon exhalation rate of the reference standard device for radon exhalation rate; $\mathrm{V}$ is volume of the radon collector, $\mathrm{m}^{3} ; \mathrm{S}$ is the base area of the radon collector, $\mathrm{m}^{2} ; \mathrm{L}$ is pumping flow rate, $\mathrm{m}^{3} / \mathrm{s}$.

\subsection{Theoretical Calculation}

According to formula (2), when the pumping rate $\mathrm{L}$ and the exhalation rate of standard source $\mathrm{P}$ are constant, the change rate of the radon concentration in radon collector $d c / d t$ decreases with the increase of radon concentration $\mathrm{C}$ in radon collector,. When the radon concentration in radon collector remain unchanged, that is $d c / d t=0$, the radon concentration $\mathrm{C}$ rises to $\mathrm{PS}-\mathrm{Lc}=0$ when the radon concentration in radon collector can reach equilibrium, the influence of the volume $\mathrm{V}$, the base area $\mathrm{S}$ and pumping flow rate $\mathrm{L}$ on equilibrium radon concentration $\mathrm{C}$ and the equilibrium time of radon concentration can be calculated separately.

\subsubsection{Effect of Radon Collector Volume on the Equilibrium Time of Radon Concentration}

Formula (3) shows that when the measurement time reaches a certain value, the radon concentration does not change any more, which is the equilibrium time of radon concentration and the radon concentration at this time is the equilibrium radon concentration. 
If $\mathrm{e}^{-\mathrm{Lt} / \mathrm{V}} \leq 1 \times 10^{-6}$ is defined as $\mathrm{e}^{-\mathrm{Lt} / \mathrm{V}}<<1$, then the equilibrium time of radon concentration is:

$$
\mathrm{t}=-\frac{V \ln \left(1 \times 10^{-6}\right)}{L}
$$

The parameter is set to $L=1 \times 10^{-5} \mathrm{~m}^{3} / \mathrm{s}$, and the variation trend of the radon concentration equilibrium time $t$ caused by different volume of radon collector is calculated according to formula (4), as shown in figure 3.

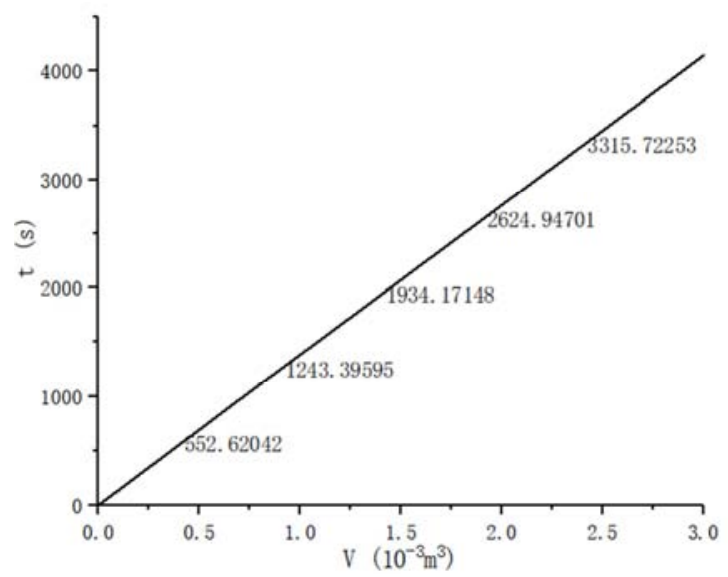

Figure 3. The Variation Trend of the Radon Concentration Equilibrium Time $t$ with the Change of the Volume of Radon Collector.

As can be seen from the trend shown in Figure 3, the time of reaching the equilibrium radon concentration increases linearly with the volume of the collector. When the volume of the radon collector exceeds $2.7 \times 10-3 \mathrm{~m}^{3}$, the equilibrium time of the radon concentration $\mathrm{t}$ will be more than $1 \mathrm{~h}$, and the accumulation time is relatively long, which is not conducive to the rapid measurement of the equilibrium radon concentration in the collector. The equilibrium time of the radon concentration should be as short as possible without affecting the measurement accuracy. The optimal equilibrium time of the radon concentration $t$ should be about 1 hour $(0.5-1 \mathrm{~h})$ based on the general requirements for radon detector and radon exhalation rate.

\subsubsection{The Influence of Radon Collector Base Area on Equilibrium Radon Concentration c}

According to formula (2), when $\mathrm{e}^{-\mathrm{Lt} / \mathrm{V}}<<1$, the radon concentration in radon collector reaches the maximum, i.e. the equilibrium radon concentration, which can be expressed by formula (5):

$$
\mathrm{c}=\frac{P S}{L}
$$

The parameter is set to $L=1 \times 10^{-5} \mathrm{~m}^{3} / \mathrm{s}$, and the reference value of the reference standard device for radon exhalation rate is $P=0.39 \mathrm{~Bq} \cdot \mathrm{m}^{-2} \cdot \mathrm{s}^{-1}$. The variation trend of equilibrium radon concentration $\mathrm{C}$ with the change of the base area of radon collector $\mathrm{S}$ is shown in Figure 4.

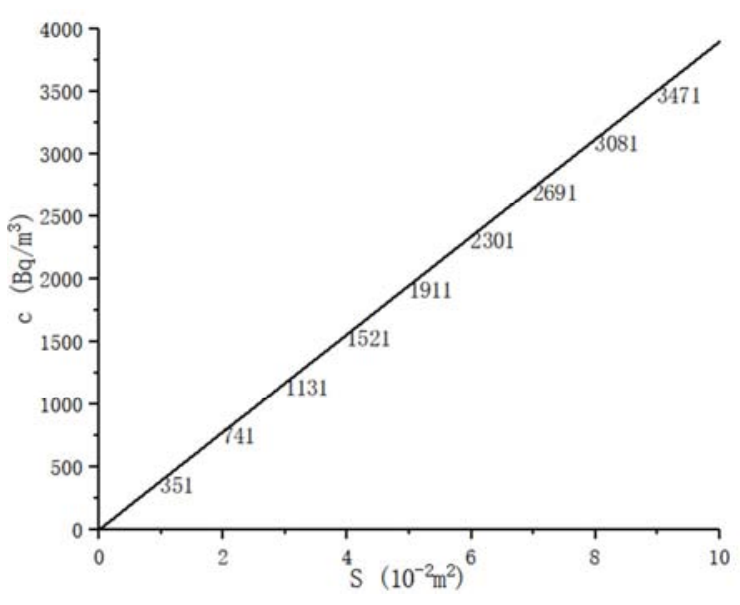

Figure 4. The Variation Trend of Equilibrium Radon Concentration C with the Change of the Base Area of Radon Collector $S$.

It can be seen from Figure 4 that the equilibrium radon concentration increases linearly with the base area of radon collector when the pumping flow rate and radon exhalation rate remain unchanged. When the base area of radon collector is $2.6 \times 10^{-2} \mathrm{~m}^{2}$, the equilibrium radon concentration in radon collector is $1014 \mathrm{~Bq} \cdot \mathrm{m}^{-3}$, and the base area of radon collector is $3.8 \times 10^{-2} \mathrm{~m}^{2}$, the equilibrium radon concentration in radon collector is $1482 \mathrm{~Bq} \cdot \mathrm{m}^{-3}$, which is a concentration range applicable to the measurement of general radon detector, because this is basically a median concentration of the measurement of radon detector, and it is also a range of concentration in which the radon detector can measure accurately. However, the radon concentration less than $1000 \mathrm{~Bq} \cdot \mathrm{m}^{-3}$ will cause large errors in the measured data of RAD7, so it is not necessary to consider the actual operation. In addition, in the measurement of radon exhalation rate on the surface of materials through open-loop mode, fresh air is pumped into the radon collector, thus the radon background in the environment should be taken into consideration. The concentration of radon background in the environment is about several to tens of $\mathrm{Bq}$. In practice, in order to make the experimental operation simple and the measurement results more accurate, fresh nitrogen is used instead of fresh air.

\subsubsection{Effect of Pumping Flow Rate on Equilibrium Radon Concentration $c$ and Radon Concentration Equilibrium Time $t$}

One of the most important parameters for measuring radon exhalation rate through double-chamber open-loop system is the pumping flow rate of the pump, which not only affects the equilibrium radon concentration of radon collector, but also affects the radon concentration equilibrium time. According to the permanent flow rate of the built-in pump and the external pump of the actual radon meter, the pumping rate of $0.1 \mathrm{~L} / \mathrm{min}$, $0.2 \mathrm{~L} / \mathrm{min}, 0.4 \mathrm{~L} / \mathrm{min}, 0.6 \mathrm{~L} / \mathrm{min}, 1.0 \mathrm{~L} / \mathrm{min}, 1.5 \mathrm{~L} / \mathrm{min}, 2.0 \mathrm{~L} / \mathrm{min}$ and $3.0 \mathrm{~L} / \mathrm{min}$ are selected to calculate.

Based on formula (4) and (5), the reference value of the radon exhalation rate of reference standard device is set up to $P=0.39 \mathrm{~Bq} \cdot \mathrm{m}^{-2} \cdot \mathrm{s}^{-1}$. The variation trend of equilibrium radon concentration $\mathrm{C}$ and radon concentration equilibrium time $\mathrm{t}$ in 
radon collectors with different base areas and volume spaces is shown in Figure 5.

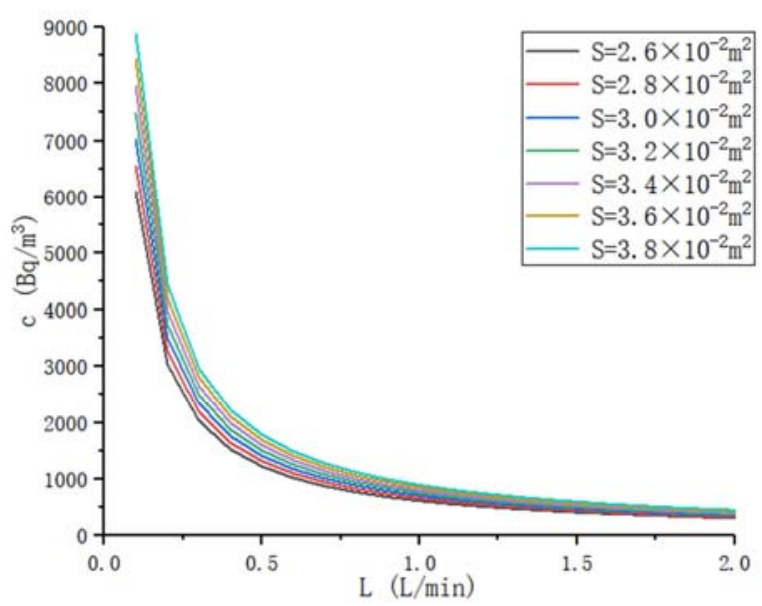

(a)

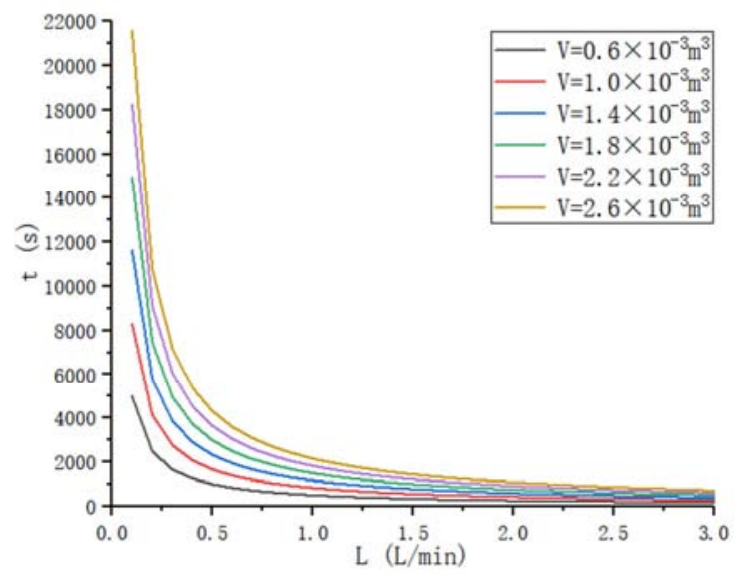

(b)

Figure 5. The Variation Trend of Equilibrium Radon Concentration $C$ and Radon Concentration Equilibrium Time $t$ in Radon Collector with Different Base Area and Volume Space.

Figure 5 shows that the equilibrium radon concentration and the radon concentration equilibrium time in radon collector decrease rapidly with the increase of the pumping flow rate when the volume and base area of radon collector remain unchanged. In the actual measurement, when the radon concentration in radon collector is below $1000 \mathrm{~Bq} / \mathrm{m}^{3}$, the measurement error of RAD7 radon detector is relatively large. It is found from the image that when the pumping flow rate is greater than $0.7 \mathrm{~L} / \mathrm{min}$, the radon concentration in radon collector is almost all below $1000 \mathrm{~Bq} / \mathrm{m}^{3}$. Therefore, the pumping flow rate under the condition of base area and radon exhalation rate for this time needs to be controlled below $0.7 \mathrm{~L} / \mathrm{min}$. Considering the radon background of air flow introduced in open-loop measurement, the equilibrium radon concentration should be more than $1000 \mathrm{~Bq} / \mathrm{m}^{3}$, but the excessively high radon concentration will increase the equilibrium time, which is not conducive to rapid measurement. Therefore, the appropriate equilibrium radon concentration should be between $1000 \mathrm{~Bq} / \mathrm{m}^{3}$ and $1500 \mathrm{~Bq} / \mathrm{m}^{3}$, and the appropriate radon concentration equilibrium time should be less than $1 \mathrm{~h}$, and the appropriate pumping flow rate should be $0.2 \mathrm{~L} / \mathrm{min}$ to $1 \mathrm{~L} / \mathrm{min}$. In order to further verify the accuracy of theoretical calculation and observe the variation of radon concentration in radon collector over time, the formula was simulated by using the Simulink simulation function of MATLAB software.

\section{Simulation Calculation by MATLAB}

\subsection{Simulation of the Influence of Radon Collector Volume and Base Area on Equilibrium Radon Concentration}

The parameter is set to $L=6 \times 10^{-4} \mathrm{~m}^{3} \cdot \mathrm{min}^{-1}$, i.e. $1 \times 10^{-5} \mathrm{~m}^{3} / \mathrm{s}$ and the reference value of radon exhalation rate for the reference standard device is set to $0.39 \mathrm{~Bq} \cdot \mathrm{m}^{-2} \cdot \mathrm{s}^{-1}$, The response from the circulating system obtained by changing the volume $\mathrm{V}$ and bottom area $\mathrm{S}$ of radon collector is shown in Figure 6.

From the data presented Figure 6, the variation trend and pattern of radon concentration in radon collector are the same when the pumping flow rate does not change. The radon concentration in radon collector tends to be constant after reaching a certain value through a logarithmic curve growth. In addition, the equilibrium radon concentration and radon concentration equilibrium time obtained from the simulation figure are in a good conformity with the theoretical calculation.

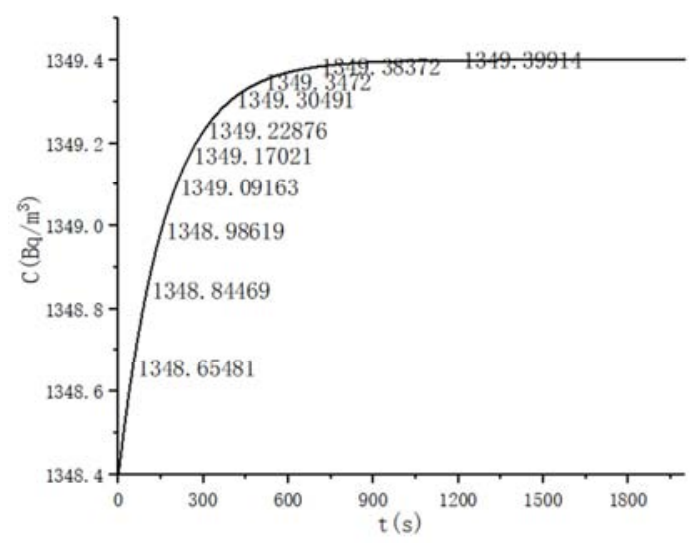

(a) $\mathrm{V}=1.7 \times 10^{-3} \mathrm{~m}^{3}, \mathrm{~S}=3.46 \times 10^{-2} \mathrm{~m}^{2}$

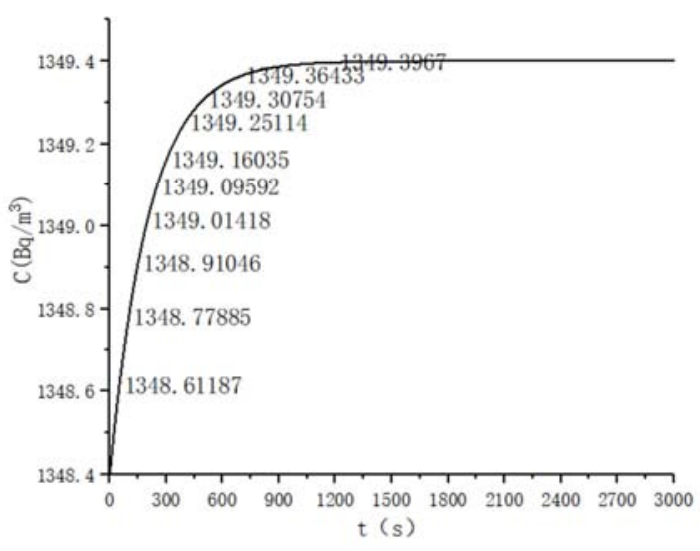

(b) $\mathrm{V}=2.1 \times 10^{-3} \mathrm{~m}^{3}, \mathrm{~S}=3.46 \times 10^{-2} \mathrm{~m}^{2}$ 


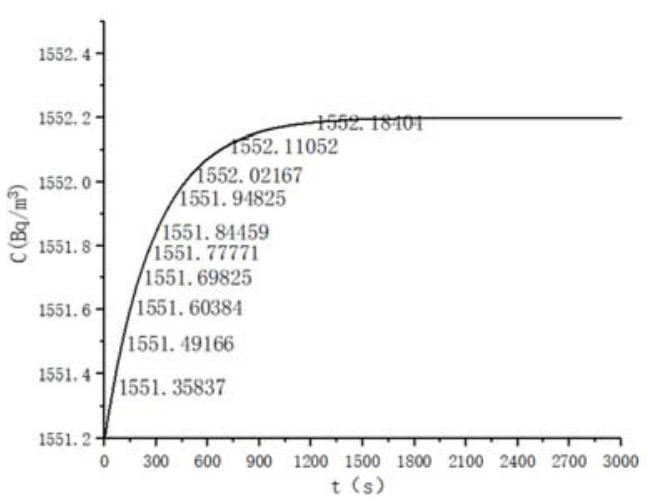

(c) $\mathrm{V}=2.9 \times 10^{-3} \mathrm{~m}^{3}, \mathrm{~S}=3.98 \times 10^{-2} \mathrm{~m}^{2}$

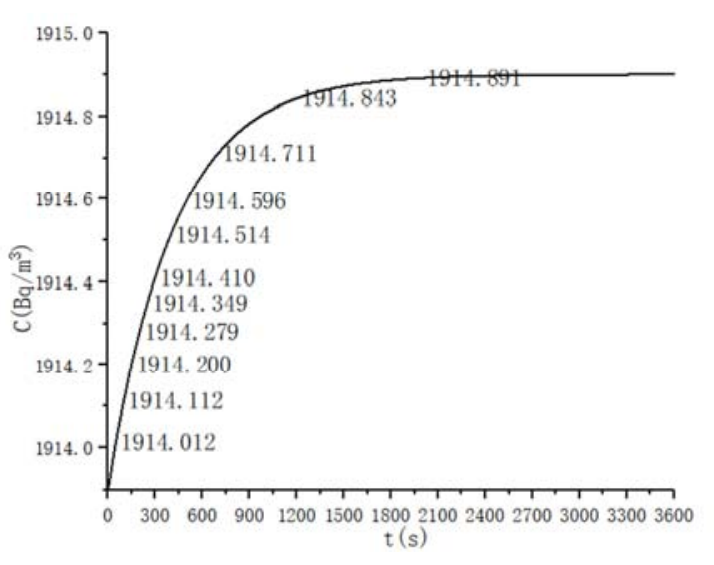

(d) $\mathrm{V}=4.2 \times 10^{-3} \mathrm{~m}^{3}, \mathrm{~S}=4.91 \times 10^{-2} \mathrm{~m}^{2}$

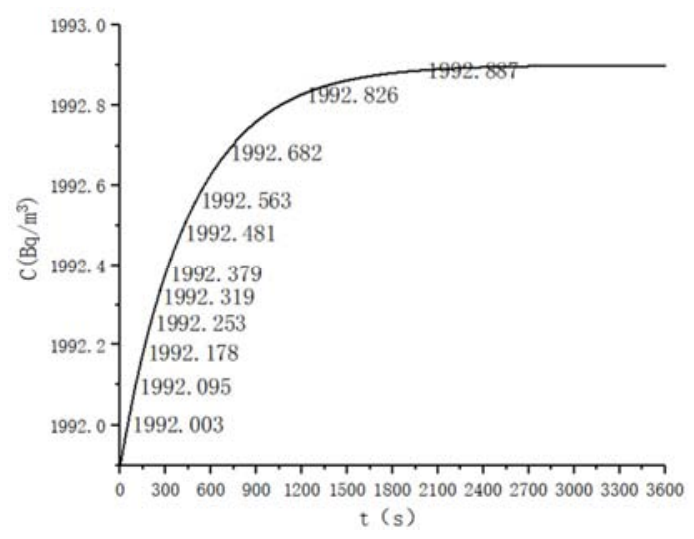

(e) $\mathrm{V}=4.6 \times 10^{-3} \mathrm{~m}^{3}, \mathrm{~S}=5.11 \times 10^{-2} \mathrm{~m}^{2}$

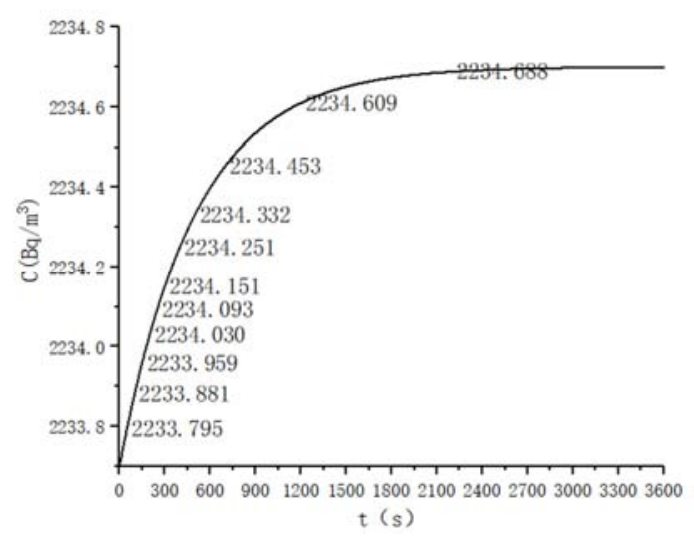

(f) $V=5 \times 10^{-3} \mathrm{~m}^{3}, \mathrm{~S}=5.73 \times 10^{-2} \mathrm{~m}^{2}$

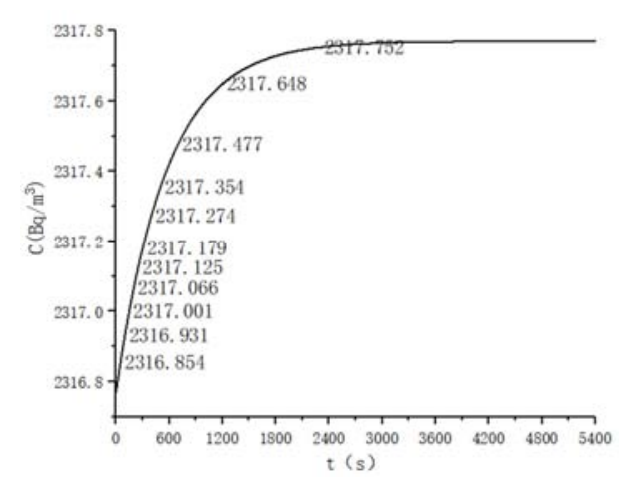

(g) $V=5.7 \times 10^{-3} \mathrm{~m}^{3}, \mathrm{~S}=5.94 \times 10^{-2} \mathrm{~m}^{2}$

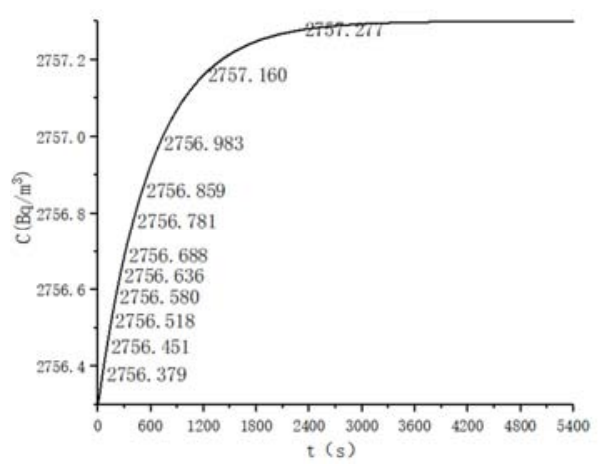

(h) $\mathrm{V}=6.1 \times 10^{-3} \mathrm{~m}^{3}, \mathrm{~S}=7.07 \times 10^{-2} \mathrm{~m}^{2}$

Figure 6. Simulation Data of Fixed Pumping Flow Rate.

\subsection{Simulation of the Influence of Pumping Flow Rate, Radon Collector Volume and Base Area on Equilibrium Radon Concentration}

The reference value of radon exhalation rate for reference standard device is set to $0.39 \mathrm{~Bq} \cdot \mathrm{m}^{-2} \cdot \mathrm{s}^{-1}$. The appropriate combination of pumping flow rate, radon collector volume and bottom area is selected for simulation calculation. The results are shown in Figure 7.

From Figure 7, it can be seen that the figure and data obtained by simulation are almost consistent with the theoretical calculation, and these four sets of parameters are ideal measurement parameters, which provide the actual experimental operation with a more accurate guidance.

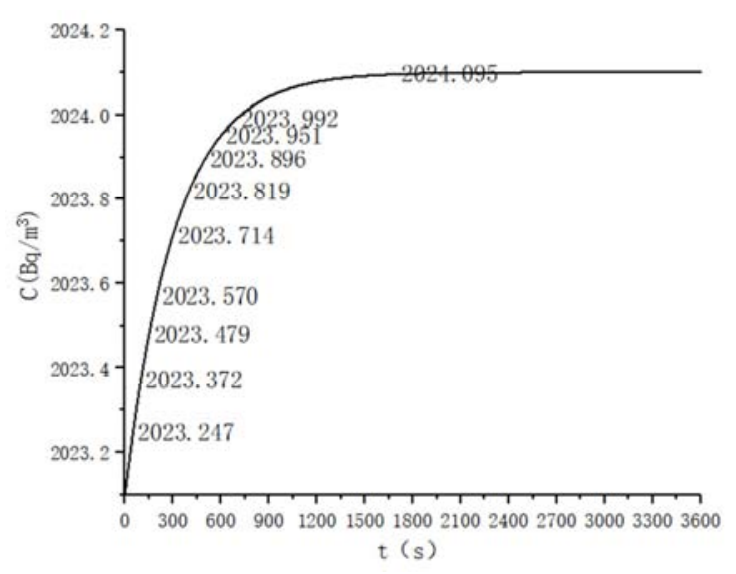

(a) $\mathrm{L}=0.4 \mathrm{~L} / \mathrm{min}, \mathrm{V}=2.1 \times 10^{-3} \mathrm{~m}^{3}, \mathrm{~S}=3.46 \times 10^{-2} \mathrm{~m}^{2}$ 


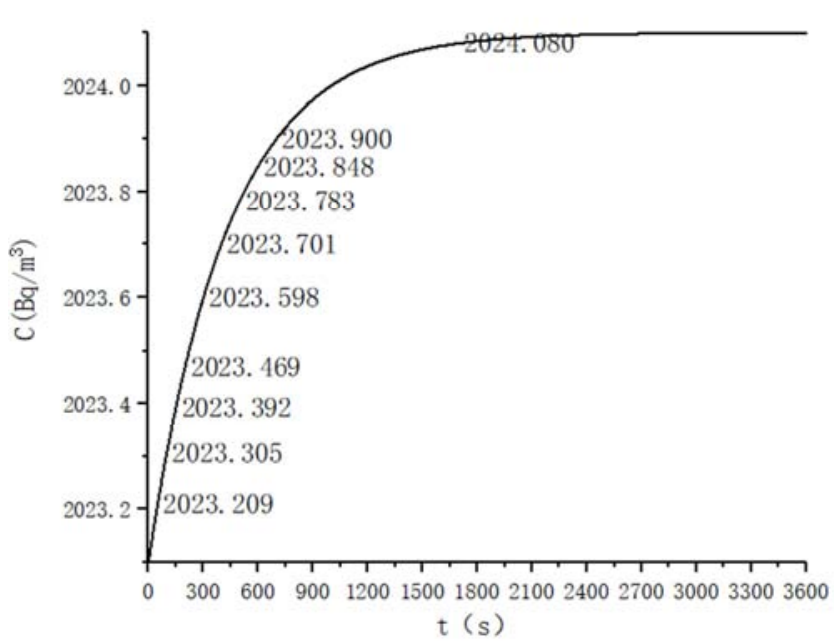

(b) $\mathrm{L}=0.4 \mathrm{~L} / \mathrm{min}, \mathrm{V}=2.9 \times 10^{-3} \mathrm{~m}^{3}, \mathrm{~S}=3.46 \times 10^{-2} \mathrm{~m}^{2}$

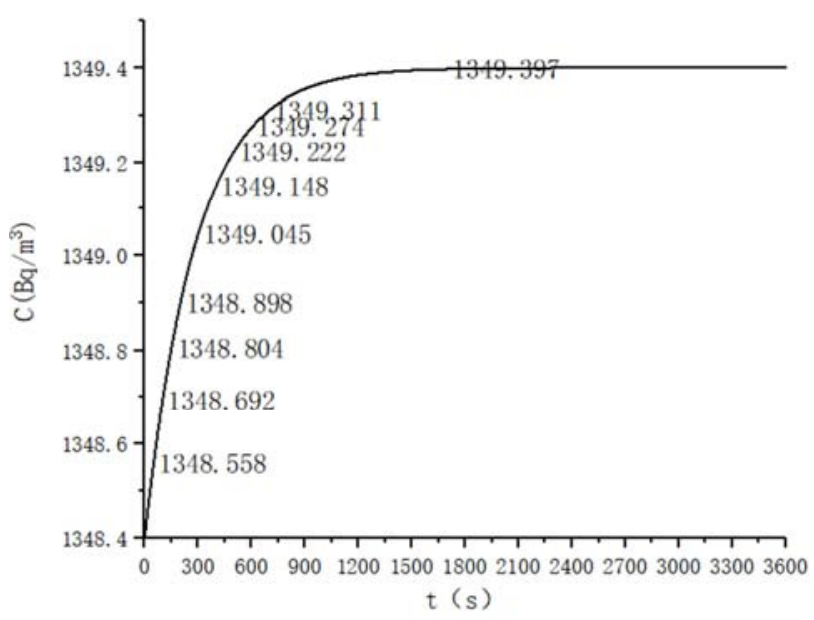

(c) $\mathrm{L}=0.6 \mathrm{~L} / \mathrm{min}, \mathrm{V}=2.9 \times 10^{-3} \mathrm{~m}^{3}, \mathrm{~S}=3.46 \times 10^{-2} \mathrm{~m}^{2}$

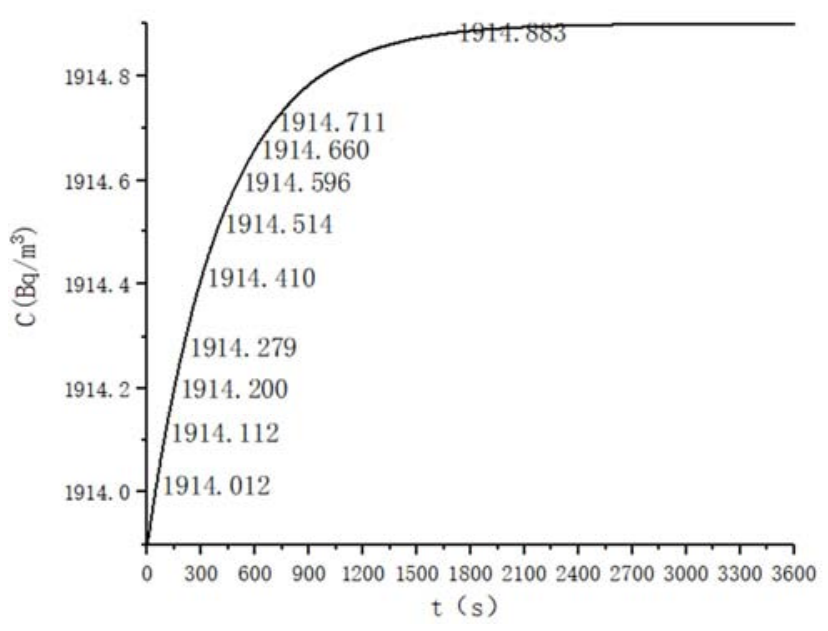

(d) $\mathrm{L}=0.6 \mathrm{~L} / \mathrm{min}, \mathrm{V}=4.2 \times 10^{-3} \mathrm{~m}^{3}, \mathrm{~S}=4.91 \times 10^{-2} \mathrm{~m}^{2}$

Figure 7. Data of the Simulation of Impact.

\subsection{Simulated Calculation of Optimum Parameters Combination}

Because of the requirement for measurement error of radon meter, the best equilibrium radon concentration is between $1000 \mathrm{~Bq} / \mathrm{m}^{3}$ and $1500 \mathrm{~Bq} / \mathrm{m}^{3}$, and the best measurement time is within 1 hour and the smaller the better. At the same time, the best pumping flow rate is between $0.4 \mathrm{~L} / \mathrm{min}$ and $1 \mathrm{~L} / \mathrm{min}$. According to formula (3), (4), (5), the parameters need to meet the following conditions:

$$
\begin{aligned}
& 1000 \leq \mathrm{c}=\frac{\mathrm{PS}}{\mathrm{L}} \leq 1500 \\
& 0<\mathrm{t}=-\frac{V \ln \left(1 \times 10^{-6}\right)}{L} \leq 3600 \\
& 6.67 \times 10^{-6} \leq L \leq 1.67 \times 10^{-5}
\end{aligned}
$$

Considering the portability, of the actual device and fast and accurate measurement of radon exhalation rate, the radon collector needs to be as small as possible and the radon concentration equilibrium time should be as short as possible without affecting the accuracy of measurement. However, in practice, the DURRIDGE RAD7 Radon Delecter (Abbreviated as RAD7) radon detector will extract the gas $(0.7 \mathrm{~L})$ from the radon collector in the measurement, which means that the volume of the radon collector must be larger than that of the gas pumped by the radon detector. In practice, over-high radon collector will lead to uneven gas in the radon collector, so the height should be less than the diameter of the bottom. The final parameters range can be obtained by optimization calculation:

$$
\begin{aligned}
& 1000 \leq \mathrm{c}=\frac{\mathrm{PS}}{\mathrm{L}} \leq 1500 \\
& 0<\mathrm{t}=-\frac{V \ln \left(1 \times 10^{-6}\right)}{L} \leq 3600 \\
& 6.67 \times 10^{-6} \leq L \leq 1.67 \times 10^{-5} \\
& V>0.0001 \\
& 0<H=\frac{V}{S} \leq 2(S / \pi)^{1 / 2}
\end{aligned}
$$

Among them, $\mathrm{C}$ is the equilibrium radon concentration, $\mathrm{Bq} / \mathrm{m}^{3} ; \mathrm{t}$ is the radon concentration equilibrium time, $\mathrm{s} ; \mathrm{P}$ is the radon exhalation rate of the reference standard device, $\mathrm{Bq} \cdot \mathrm{m}^{-2} \cdot \mathrm{s}^{-1} ; \mathrm{V}$ is the volume of radon collector, $\mathrm{m}^{3} ; \mathrm{S}$ is the base area of the radon collector, $\mathrm{m}^{2}$; $\mathrm{L}$ is the pumping flow rate, $\mathrm{m}^{3} / \mathrm{s}$.

Under the conditions designed by this study, the radon exhalation rate of the reference standard device is set to be $0.39 \mathrm{~Bq} \cdot \mathrm{m}^{-2} \cdot \mathrm{s}^{-1}$, and the data simulated by MATLAB through the selection of different volumes and bottom areas of radon collector, which is shown in Figure 8. In the four groups of simulation, the minimum radon concentration equilibrium time in a is $2151 \mathrm{~s}$, and other parameters are within the designed range. Therefore, the optimum pumping flow rate for measuring radon exhalation rate of $0.39 \mathrm{~Bq} \cdot \mathrm{m}^{-2} \cdot \mathrm{s}^{-1}$ is $1.349 \times 10^{-5} \mathrm{~m}^{3} / \mathrm{s}$ using radon collector with volume of $2.1 \times 10^{-3} \mathrm{~m}^{3}$ and base area of $3.46 \times 10^{-2} \mathrm{~m}^{-2}$. This method can also be used to obtain the best measurement parameters for radon exhalation rate under different conditions. 


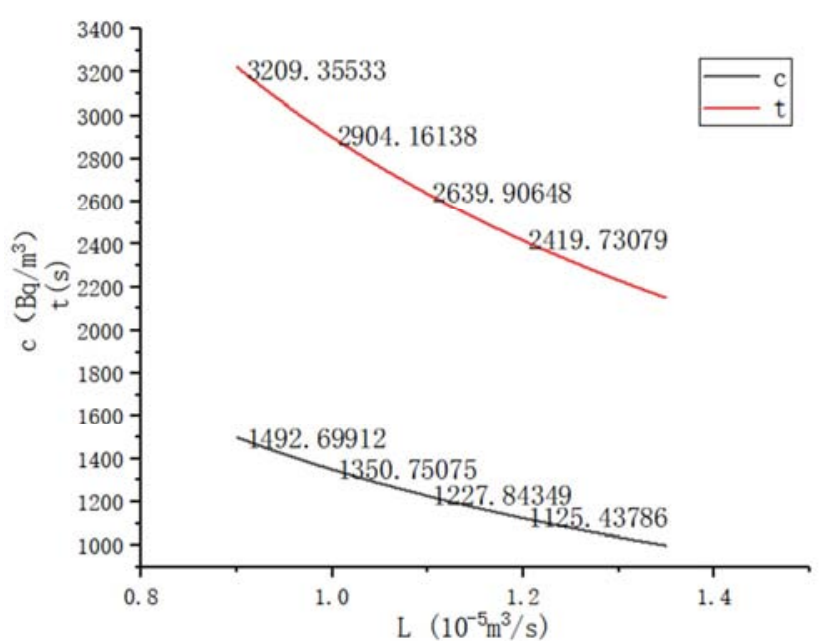

(a) $\mathrm{V}=2.1 \times 10^{-3} \mathrm{~m}^{3}, \mathrm{~S}=3.46 \times 10^{-2} \mathrm{~m}^{2}$

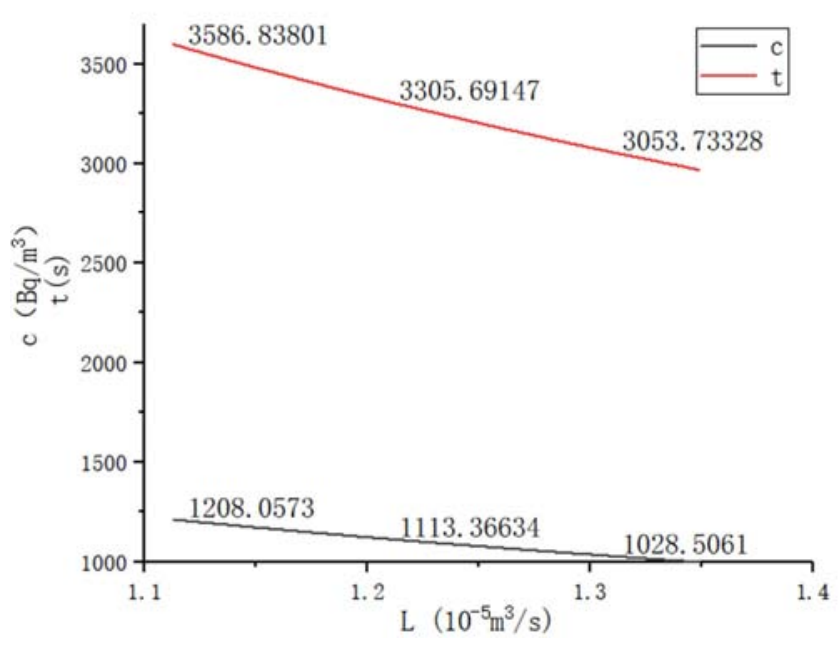

(b) $\mathrm{V}=2.9 \times 10^{-3} \mathrm{~m}^{3}, \mathrm{~S}=3.46 \times 10^{-2} \mathrm{~m}^{2}$

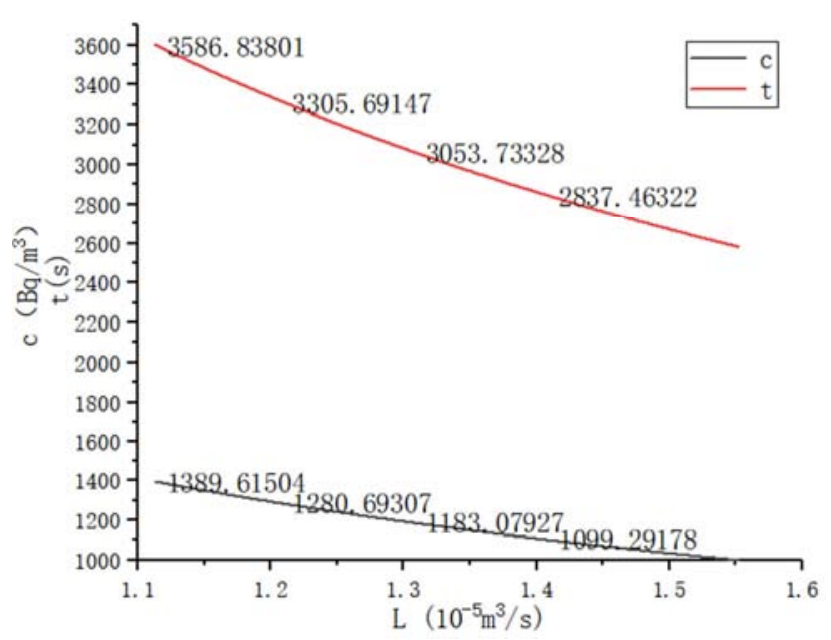

(c) $\mathrm{V}=2.9 \times 10^{-3} \mathrm{~m}^{3}, \mathrm{~S}=3.98 \times 10^{-2} \mathrm{~m}^{2}$

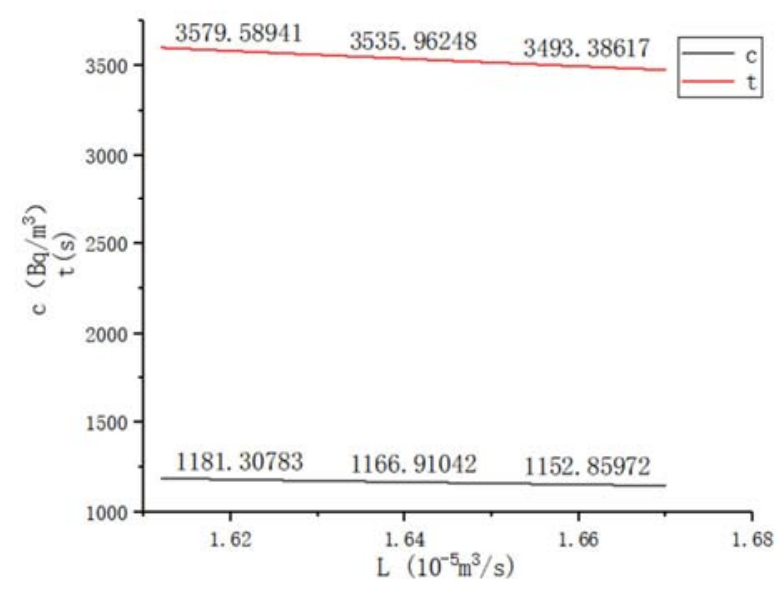

(d) $\mathrm{V}=4.2 \times 10^{-3} \mathrm{~m}^{3}, \mathrm{~S}=4.91 \times 10^{-2} \mathrm{~m}^{2}$

Figure 8. Simulated Impact Data Diagram.

\section{Experimental Measurement of Radon Exhalation Rate}

In this experiment, a double-chamber open-loop electrostatic absorption method was used to measure radon exhalation rate. The radon exhalation rate of the standard device is $0.70 \mathrm{~Bq} \cdot \mathrm{m}^{-2} \cdot \mathrm{s}^{-1}$. According to the theoretical optimization calculation, four suitable radon collectors are selected: $2100 \mathrm{~mL}$ single inlet radon collector, $2100 \mathrm{~mL}$ four inlets radon collector, $5700 \mathrm{~mL}$ single inlet radon collector and $5700 \mathrm{~mL}$ four inlets radon collector. The measured data are shown in Figure 9 and Tables 1-4. The a-d in Figure 9 corresponds to Tables 1-4.

From a, b of Figure 9 and Tables 1 and 2, it can be seen that the radon concentration in radon collector increases exponentially over time on the whole. The time of reaching equilibrium radon concentration in radon collector is within 22-26 minutes, which is in a good conformity with the theoretical value. The equilibrium radon concentration in radon collector in the experiment is $2900-3100 \mathrm{~Bq} / \mathrm{m}^{3}$ and $2600-2800$ $\mathrm{Bq} / \mathrm{m}^{3}$ respectively and the four inlets are in a good conformity with the theoretical values, which indicates that the uniformity of air flow in radon collector will have some influence on the experimental results. Both groups of data fluctuated after reaching equilibrium radon concentration in radon collector. This may be due to the instability of the pumping flow rate inside the radon meter, resulting in fluctuations in data.

From the c, d of Figure 9 and Tables 3 and 4, it can be seen that the radon concentration in radon collector increases exponentially over time on the whole. The equilibrium radon concentration time in radon collector happens at about 60 minutes, which is in a good consistency with the theoretical value. In the experiment, the equilibrium radon concentration in the radon collector is about $4800-5000 \mathrm{~Bq} / \mathrm{m}^{3}$ and $4300-4500 \mathrm{~Bq} / \mathrm{m}^{3}$ respectively. The equilibrium radon concentration in the radon collector with four air inlets conforms better with the theoretical value compared with that in the radon collector with single air inlet. 


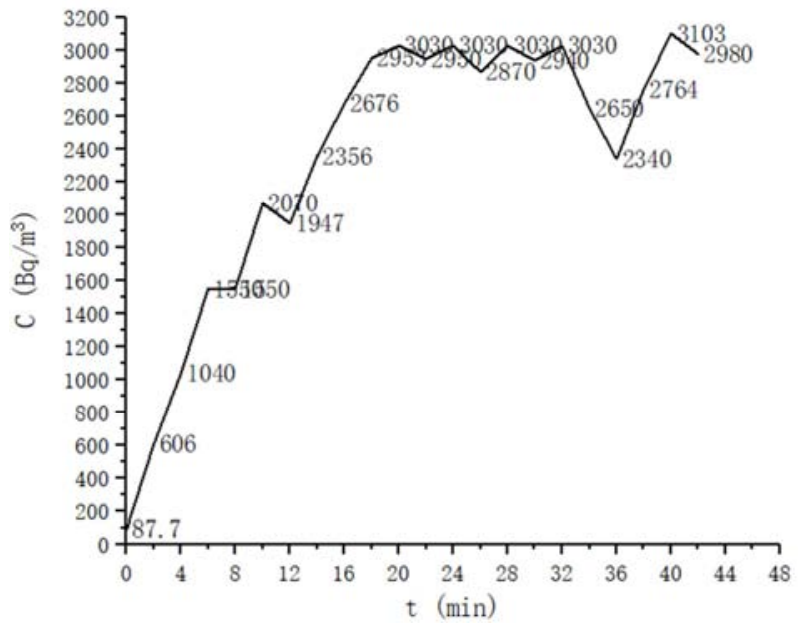

(a)

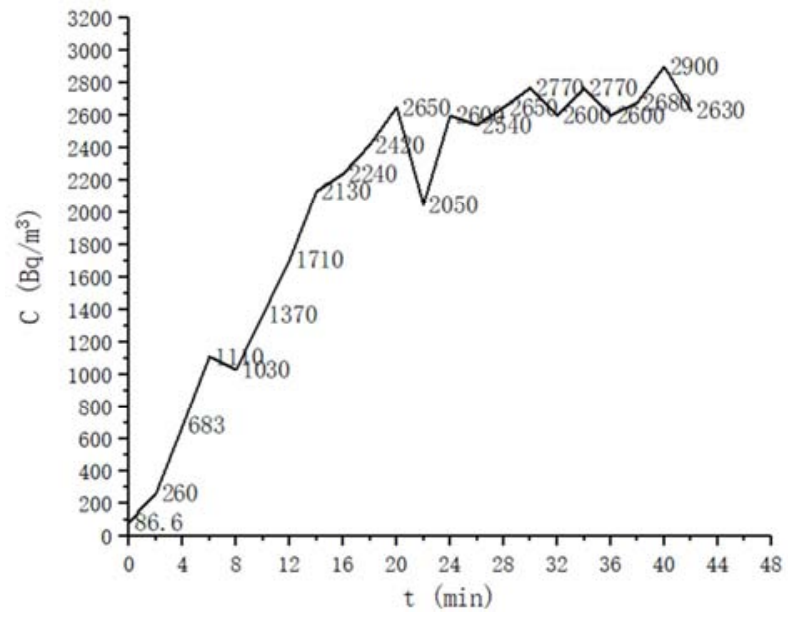

(b)

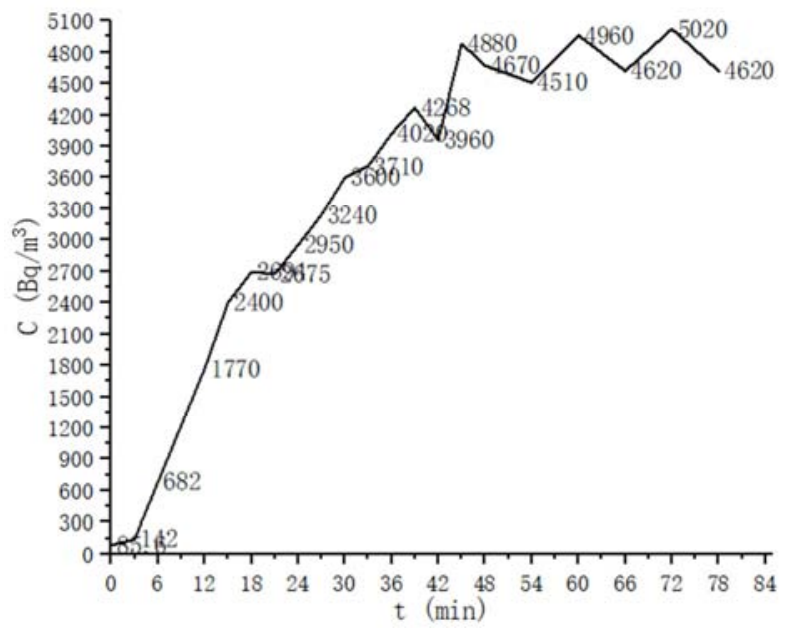

(c)

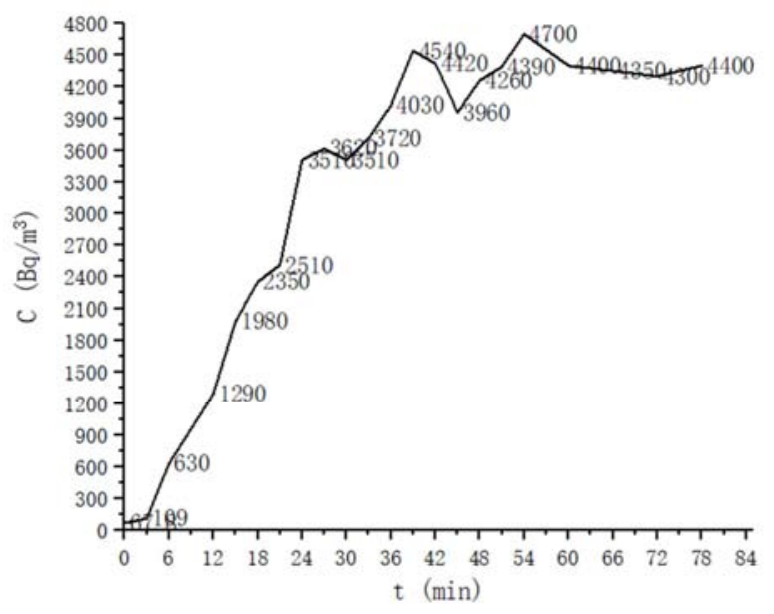

(d)

Figure 9. Response of Radon Concentration in Radon Collector to Time.

Table 1. Parameters of $2100 \mathrm{~mL}$ Radon Collector with Single Air Inlet.

\begin{tabular}{llll}
\hline Parameter & Data & Parameter & Data \\
\hline Pumping Rate & $0.6 \mathrm{~L} / \mathrm{min}$ & Temperature & $28^{\circ} \mathrm{C}$ \\
Theoretical Equilibrium Radon Concentration & $2422 \mathrm{~Bq} / \mathrm{m}^{3}$ & Relative Humidity & $13 \%$ \\
Theoretical Equilibrium Radon Concentration Time & $24 \mathrm{~min}$ & P (Working Current) & $80 \mathrm{~mA}$ \\
Radon Background & $87.7 \mathrm{~Bq} / \mathrm{m}^{3}$ & B (Working Voltage) & $6.20 \mathrm{~V}$ \\
Base area & $21 \mathrm{~cm} \times 17 \mathrm{~cm}$ & Volume & $2100 \mathrm{~mL}$ \\
\hline
\end{tabular}

Table 2. Parameters of $2100 \mathrm{~mL}$ Radon Collector with Four Air Inlets.

\begin{tabular}{llll}
\hline Parameter & Data & Parameter & Data \\
\hline Pumping rate & $0.6 \mathrm{~L} / \mathrm{min}$ & temperature & $27.4^{\circ} \mathrm{C}$ \\
Theoretical Equilibrium Radon Concentration & $2422 \mathrm{~Bq} / \mathrm{m}^{3}$ & Relative Humidity & $13 \%$ \\
Theoretical Equilibrium Radon Concentration Time & $24 \mathrm{~min}$ & P (Working Current) & $80 \mathrm{~mA}$ \\
Radon Background & $86.6 \mathrm{~Bq} / \mathrm{m}^{3}$ & B (Working Voltage) & $6.20 \mathrm{~V}$ \\
Base area & $21 \mathrm{~cm} \times 17 \mathrm{~cm}$ & Volume & $2100 \mathrm{~mL}$ \\
\hline
\end{tabular}

Table 3. Parameters of $5700 \mathrm{~mL}$ Radon Collector with Single Air Inlet.

\begin{tabular}{llll}
\hline Parameter & Data & Parameter & Data \\
\hline Pumping rate & $0.6 \mathrm{~L} / \mathrm{min}$ & Temperature & $27.6^{\circ} \mathrm{C}$ \\
Theoretical Equilibrium Radon Concentration & $4310 \mathrm{~Bq} / \mathrm{m}^{3}$ & Relative Humidity & $12 \%$ \\
Theoretical Equilibrium Radon Concentration Time & $65 \mathrm{~min}$ & P (Working Current) & $90 \mathrm{~mA}$ \\
Radon Background & $56.8 \mathrm{~Bq} / \mathrm{m}^{3}$ & B (Working Voltage) & $6.36 \mathrm{~V}$ \\
Base area & $27.5 \mathrm{~cm} \times 23 \mathrm{~cm}$ & Volume & $5700 \mathrm{~mL}$ \\
\hline
\end{tabular}


Table 4. Parameters of $5700 \mathrm{~mL}$ Radon Collector with Four Air Inlets.

\begin{tabular}{lll}
\hline Parameter & Data & Parameter \\
\hline Pumping rate & $0.6 \mathrm{~L} / \mathrm{min}$ & Temperature \\
Theoretical Equilibrium Radon Concentration & $4310 \mathrm{~Bq} / \mathrm{m}^{3}$ & Relative Humidity \\
Theoretical Equilibrium Radon Concentration Time & $65 \mathrm{~min}$ & P (Working Currentt \\
Radon background & $57.4 \mathrm{~Bq} / \mathrm{m}^{3}$ & B (Working Voltage) \\
Bottom area & $27.5 \mathrm{~cm} \times 23 \mathrm{~cm}$ & Volume \\
\hline
\end{tabular}

\section{Conclusion}

Through experimental and theoretical verification, it has been found that the radon concentration in radon collector increases exponentially over time. Under the condition of constant pumping flow rate, the radon concentration equilibrium time in radon collector increases linearly with the volume $\mathrm{V}$ of radon collector, and the equilibrium radon concentration in radon collector only presents proportional relationship with base area $\mathrm{S}$ of radon collector; when the volume and base area of radon collector are unchanged, the equilibrium radon concentration and radon concentration equilibrium time in radon collector are in inversely proportional relationship with the increase of pumping flow rate. Through theoretical calculation and experiment verification, the radon concentration equilibrium time in radon collector is inversely proportional to the increase of pumping rate. The measurement parameters of radon exhalation rate measurement can be optimized according to the relationship formula as follows:

$$
\begin{aligned}
& 1000 \leq \mathrm{c}=\frac{\mathrm{PS}}{\mathrm{L}} \leq 1500 \\
& 0<\mathrm{t}=-\frac{V \ln \left(1 \times 10^{-6}\right)}{L} \leq 3600 \\
& 6.67 \times 10^{-6} \leq L \leq 1.67 \times 10^{-5} \\
& V>0.0001 \\
& 0<H=\frac{V}{S} \leq 2(S / \pi)^{1 / 2}
\end{aligned}
$$

The radon collector with four air inlets was adopted in the actual measurement, which ensures the gas uniformity in the radon collector and reduces the errors caused by the non-uniformity of the gas in the measurement. Through the optimization analysis of this study, the efficiency and accuracy of radon exhalation rate measurement are greatly improved with the optimum parameters of radon collector for measuring radon exhalation rate of $0.39 \mathrm{~Bq} \cdot \mathrm{m}^{-2} \cdot \mathrm{s}^{-1}$ obtained: volume $2.1 \times 10^{-3} \mathrm{~m}^{3}$, base area $3.46 \times 10^{-2} \mathrm{~m}^{-2}$, pumping flow rate $1.349 \times 10^{-5} \mathrm{~m}^{3} / \mathrm{s}$. This method can also be used to obtain the best measurement parameters of radon exhalation rate under different conditions.

\section{References}

[1] UNSCEAR. Sources and effects of ionzing radiation [R]. Sweden UNSCEAR, 2000.

[2] Chen Ling, Pan Ziqiang, Liu Lin, et al. Preliminary investigation and study of ${ }^{222} \mathrm{Rn}$ and ${ }^{220} \mathrm{Rn}$ levels in China's underground coal mines [N]. Annual Report of China Institute of Atomic Energy, 2005: 234-235.
[3] Guogang Jia, M. Belli, M. Blasi, et al. Determination of $210 \mathrm{~Pb}$ and 210Po in mineral and biological environmental samples [J]. Journal of Radioanalytical and Nuclear Chemistry, 2001, 247 (3): 491-499.

[4] Shang Bing, He Qinghua, Wang Zuoyuan, Zhu Changshou. Study on the level of indoor radon action in China [J]. Chinese Journal of Radiological Medicine and Protection, 2003, 23 (6): 462-465.

[5] Xiao Detao, Zhao Guizhi, Xiao Yongjun, Ma Guangnao. ${ }^{220} \mathrm{Rn}$ cumulative measurement of absorption volume method and its application [J]. Nuclear Techniques, 2005, 28 (9): 688-692.

[6] Zhao Changyou. Thorium and Uranium in Bayan Obo [J]. Rare Earth Information. 2006, (7): 12-15.

[7] Xiao Detao, Liang Ganzhuang, Zhao Guizhi, Lingqiu. ${ }^{220} \mathrm{Rn}$ 's influence on the measurement of radon exhalation rate [J]. Atomic Energy Science and Technology, 2002, 36 (6): 543-547.

[8] Zhang Qiang, Deng Yuequan, Dong Faqin, Xu Guangliang, Yang Rui, He Dengliang. Research status and Prospect of radon radiation pollution and protection of industrial waste base building materials [J]. Materials Review, 2007, 21 (10): 79-83.

[9] Chen Ling, Xie Jianlun, Huanglong. Measurement of radon surface emission rate and consideration of related factors $[\mathrm{J}]$. Radiation Protection Bulletin, 1998, 18 (6): 28-36.

[10] Yang Yaxin, Wu Xinmin, Wu Yamei, et al. Experimental study on the determination of radon exhalation rate by double filtration membrane method $[\mathrm{J}]$. Bulletin of Science and Technology, 2001, (1): 38-42.

[11] Guo Qiuju, Cheng Jianping. The measurement of ${ }^{222} \mathrm{Rn}$ and ${ }^{220} \mathrm{Rn}$ daughter levels in ambient air and soil precipitation rate in Zhuhai [J], Radiation Protection, 2004, 24 (2): 110-115.

[12] Zhang Wentao, Li Aiwu, Zhang Zhilong, Gou Quanlu. Development of REM-II Radon Exhalation Rate Instrument [J]. Nuclear Electronics \& Detection Technology, 2002, 22 (2): 149-151.

[13] Piotr Szajerski, Joanna Celinska, Henryk Bem, Andrzej Gasiorowski, Rafal Anyszka, Piotr Dziugan. Radium content and radon exhalation rate from sulfur polymer composites (SPC) based on mineral fillers [J]. Construction and Building Materials, 2019, 20 (2): 390-398.

[14] Yan Liu, Jimian Zhang, Jiulin Yuan, et al. Effect of Pressure on Radon Exhalation Rate from Medium Surface [J]. Radiation Hygiene in China, 2018, 27 (06): 54-56.

[15] Yongjun Ye, Hanguan Wen, Xiangyan Li, et al. Grey Correlation Analysis of Radon Exhalation Rate and Environmental Factors in Uranium Tailings Beach [J]. Industrial Safety and Environmental Protection, 2018, 44 (04): 100-102+107.

[16] Jagadeesha B G, Narayana Y. Effect of Grain Size on Radon Exhalation Rate in the Soils of Hassan District of Southern India [J]. Radiochemistry, 2018, 60 (3): 328-333. 